Bull. Austral. Math. Soc.

VoL. 56 (1997) [499-505]

\title{
A NOTE ON THE PERMUTATION BEHAVIOUR OF THE DICKSON POLYNOMIALS OF THE SECOND KIND
}

\section{HENDERSON}

In this note known factorisation results for the Dickson polynomials of the second kind, $f_{k}(X, a)$, are used to obtain simple restrictions on those $k$ for which $f_{k}(X, a)$ is a permutation polynomial over $\mathbb{F}_{q}$.

\section{INTRODUCTION}

Let $\mathbb{F}_{q}$ denote the finite field of order $q$ where $q=p^{e}$ and $p$ is a prime. Let $\mathbb{F}_{q}^{*}$ represent the non-zero elements of $\mathbb{F}_{q}$ and $\eta$ denote the quadratic character on $\mathbb{F}_{q}^{*}$. A polynomial $f \in \mathbb{F}_{q}[X]$ is called a permutation polynomial of $\mathbb{F}_{q}$ if the mapping induced by $f$ permutes $\mathbb{F}_{q}$. If $f \in \mathbb{F}_{q}[X]$ has no repeated roots over any extension of $\mathbb{F}_{q}$ then $f$ will be called simple. Let $a \in \mathbb{F}_{q}^{*}$ and $k$ be a positive integer. Every $x \in \mathbb{F}_{q}$ may be written as $x=u+a u^{-1}$ where $u \in \mathbb{F}_{q^{2}}$ is a root of the quadratic $Z^{2}-x Z+a$. Under these circumstances $u$ will satisfy $u^{q-1}=1$ or $u^{q+1}=a$. The Dickson polynomial of the second kind (DPSK), $f_{k}(X, a) \in \mathbb{F}_{q}[X]$, can be defined by

$$
f_{k}(x, a)=\frac{u^{k+1}-a^{k+1} u^{-(k+1)}}{u-a u^{-1}}
$$

with the condition that $u \neq \pm b$ if $a=b^{2}$ for some $b \in \mathbb{F}_{q}$. The excluded values are calculated using $f_{k}(2 b, a)=(k+1) b^{k}$ and $f_{k}(-2 b, a)=(k+1)(-b)^{k}$. Other definitions of these polynomials can be found in the monograph [5] which is devoted to Dickson polynomials of the first and second kind. Some results on the permutation behaviour of the DPSK have been found, see $[7,4]$. If $q=p$ or $q=p^{2}$ then the conditions given in [7] were shown to be both necessary and sufficient in [2] (case $q=p$ ) and [3] (case $q=p^{2}$ ).

Some factorisations of the Dickson polynomials of the first kind are contained in [5, Chapter 2]. In [1] the factorisation of both types of Dickson polynomials over a finite field was determined. The permutation properties of the Dickson polynomials of the first kind are well understood, see $[\mathbf{5}$, Chapter 2]. Here we use results from [1] to obtain simple restrictions on those $k$ for which $f_{k}(X, a)$ can be a permutation polynomial.

The factorisation of the DPSK over $\mathbb{F}_{q}$ is closely connected to the factorisation of the cyclotomic polynomials. We can use the formulas for the factorisation of $X^{k+1}-1$ into cyclotomic polynomials, see [6], to obtain similar factorisations for the DPSK.

Received 17th March, 1997.

Copyright Clearance Centre, Inc. Serial-fee code: 0004-9729/97 $\$ A 2.00+0.00$. 
LEMma 1.1. Suppose that $k+1$ is not divisible by $p$. Then the factorisation of $f_{k}(X, a)$ is given by

$$
f_{k}(x, a)=\prod_{\substack{d \mid(k+1) \\ d \neq 1}} \prod_{\substack{m=1 \\(m, d)=1}}^{d}\left(u-\zeta_{d}^{m} a u^{-1}\right)
$$

where $\zeta_{d}$ is a dth root of unity over $\mathbb{F}_{q}$.

Proof: From the definition of the DPSK we have

$$
f_{k}(x, a)=\frac{u^{k+1}-a^{k+1} u^{-(k+1)}}{u-a u^{-1}}=a^{k} u^{k}\left(\frac{\left(u^{2} a^{-1}\right)^{(k+1)}-1}{u^{2} a^{-1}-1}\right) .
$$

By substituting $y$ for $u^{2} a^{-1}$ the problem of factorising $f_{k}(x, a)$ becomes the problem of determining the factors of the polynomial $\left(y^{k+1}-1\right)(y-1)$. By using the factorisation of the cyclotomic polynomials

$$
\begin{aligned}
f_{k}(x, a) & =\frac{a^{(k+1)} u^{-(k+1)}}{u-a u^{-1}} \prod_{d \mid(k+1)} Q_{d}\left(a^{-1} u^{2}\right) \\
& =\frac{a^{(k+1)} u^{-(k+1)}}{u-a u^{-1}} \prod_{d \mid(k+1)} \prod_{\substack{m=1 \\
(m, d)=1}}^{d}\left(a^{-1} u^{2}-\zeta_{d}^{m}\right) \\
& =\left(u-a u^{-1}\right)^{-1} \prod_{d \mid(k+1)} \prod_{\substack{m=1 \\
(m, d)=1}}^{d}\left(u-\zeta_{d}^{m} a u^{-1}\right) \\
& =\prod_{\substack{d \mid(k+1) \\
d \neq 1}} \prod_{\substack{m=1 \\
(m, d)=1}}^{d}\left(u-\zeta_{d}^{m} a u^{-1}\right) .
\end{aligned}
$$

Suppose that $p^{n}$ is the largest power of $p$ dividing $k+1$. Then $k+1=m p^{n}$ for some $m \in \mathbb{Z}$ and it is a simple matter to show

$$
f_{m p^{n}-1}(X, a)=f_{m-1}^{p^{n}}(X, a)\left(X^{2}-4 a\right)^{\left(p^{n}-1\right) / 2} .
$$

We can completely factorise a DPSK over $\mathbb{F}_{q}$ using this identity and Lemma 1.1. We end this section with a simple but useful identity for the DPSK.

LEmma 1.2. Let $a, a^{\prime} \in \mathbb{F}_{q}^{*}$ satisfy $\eta\left(a a^{\prime}\right)=1$. Then $f_{k}(X, a)$ permutes $\mathbb{F}_{q}$ if and only if $f_{k}\left(X, a^{\prime}\right)$ permutes $\mathbb{F}_{q}$.

Proof: As $\eta\left(a a^{\prime}\right)=1$ then there exists $b \in \mathbb{F}_{q}^{*}$ such that $a=b a^{\prime}$. Then for $x=u+a u^{-1} \in \mathbb{F}_{q}$

$$
b^{k} f_{k}(x, a)=\frac{(b u)^{k+1}-\left(b^{2} a\right)^{k+1}(b u)^{-(k+1)}}{b u-b^{2} a(b u)^{-1}}=f_{k}\left(b x, b^{2} a\right) .
$$


It now follows that either both of the polynomials permute $\mathbb{F}_{q}$ or both do not permute $\mathbb{F}_{q}$.

\section{LiNEAR FACTORS OF THE DPSK WHERE $q$ IS ODD}

In this section we assume $q$ is odd. Lemma 1.1 leads to the next lemma concerned with linear factors of $f_{k}(X, a)$, or rather, their absence.

Lemma 2.1. Let $\eta(a)=1$. Then $f_{k}(X, a)$ has no roots in $\mathbb{F}_{q}$ if and only if $\left(k+1, p\left(q^{2}-1\right)\right)=1$.

Proof: Let $\eta(a)=1$ and suppose $f_{k}(X, a)$ has no roots in $\mathbb{F}_{q}$. Let $b \in \mathbb{F}_{q}$ satisfy $b^{2}=a$. If $p$ did divide $k+1$ then from $(1),\left(X^{2}-4 a\right)=(X-2 b)(X+2 b)$ are factors of $f_{k}(X, a)$. Suppose $(k+1, q-1)=d>1$. Then $d$ divides $q-1$ and there exist non-trivial $d$ th roots of unity in $\mathbb{F}_{q}$. If $d$ is even then -1 is a $d$ th root over $\mathbb{F}_{q}$ and from Lemma $1.1 x=u+a u^{-1}$ divides $f_{k}(x, a)$. If $d$ is odd then let $m$ be an even integer satisfying $1<m<d$ and $(m, d)=1$. Let $\zeta_{d}$ be a primitive $d$ th root of unity in $\mathbb{F}_{q}$. Then from Lemma 1.1

$$
\begin{aligned}
\left(u-\zeta_{d}^{m} a u^{-1}\right)\left(u-\zeta_{d}^{-m} a u^{-1}\right) & =\left(u+a u^{-1}\right)^{2}-a\left(\zeta_{d}^{m / 2}+\zeta_{d}^{-m / 2}\right)^{2} \\
& =\left(x+b\left(\zeta_{d}^{m / 2}+\zeta_{d}^{-m / 2}\right)\right)\left(x-b\left(\zeta_{d}^{m / 2}+\zeta_{d}^{-m / 2}\right)\right)
\end{aligned}
$$

divides $f_{k}(x, a)$ over $\mathbb{F}_{q^{2}}$. Hence $(k+1, q-1)=1$. Suppose $(k+1, q+1)=d>1$. As $(k+1, q-1)=1$ then $d$ must be odd. Since $d>1$ there are non-trivial $d$ th roots of unity in $\mathbb{F}_{q^{2}}$. Again let $m$ be an even integer satisfying $1<m<d$ and $(m, d)=1$. Let $\zeta_{d}$ be primitive $d$ th root of unity in $\mathbb{F}_{q^{2}}$. From Lemma 1.1

$$
\left(u-\zeta_{d}^{m} a u^{-1}\right)\left(u-\zeta_{d}^{-m} a u^{-1}\right)=\left(x+b\left(\zeta_{d}^{m / 2}+\zeta_{d}^{-m / 2}\right)\right)\left(x-b\left(\zeta_{d}^{m / 2}+\zeta_{d}^{-m / 2}\right)\right)
$$

divides $f_{k}(x, a)$. As $d$ divides $q+1$ then

$$
\left(\zeta_{d}^{m / 2}+\zeta_{d}^{-m / 2}\right)^{q}=\left(\zeta_{d}^{-1} \zeta_{d}^{q+1}\right)^{m / 2}+\left(\zeta_{d}^{-1} \zeta_{d}^{q+1}\right)^{-m / 2}=\zeta_{d}^{-m / 2}+\zeta_{d}^{m / 2}
$$

Therefore the divisors of $f_{k}(x, a)$ found are divisors of $f_{k}(x, a)$ over $\mathbb{F}_{q}$. Hence $(k+1$, $q+1)=1$. From these arguments we can conclude $\left(k+1, p\left(q^{2}-1\right)\right)=1$.

Conversely, let $\left(k+1, p\left(q^{2}-1\right)\right)=1$. Then for any $d$ dividing $k+1$ there are no $d$ th roots of unity in $\mathbb{F}_{q^{2}}$. Suppose that $f_{k}(X, a)$ has a linear factor. Then there is a solution $x \in \mathbb{F}_{q^{2}}$ to $f_{k}(x, a)=0$. Therefore one of the factors in Lemma 1.1 must satisfy $u-\zeta_{d}^{m} a u^{-1}=0$ for some $u \in \mathbb{F}_{q^{2}}$. By rearranging, $u^{2} a^{-1}=\zeta_{d}^{m}$. Hence $\zeta_{d}^{m}$ is an element of $\mathbb{F}_{q^{2}}$. This contradicts the observation that there are no $d$ th roots of unity in $\mathbb{F}_{q^{2}}$ for any divisor $d$ of $k+1$.

We have a similar result for non-square $a \in \mathbb{F}_{q}$. 
LEMMA 2.2. Let $\eta(a)=-1$. Then $f_{k}(X, a)$ has no roots in $\mathbb{F}_{q}$ if and only if $k+1$ is odd.

Proof: From the definition of the DPSK, $X$ is a factor of $f_{k}(X, a)$ if and only if $k+1$ is even. Suppose $k+1$ is odd and $f_{k}(X, a)$ has a non-zero root in $\mathbb{F}_{q}$. Then there is a non-zero root $u \in \mathbb{F}_{q^{2}}$ to $u^{2(k+1)}=a^{k+1}$ where either $u^{q-1}=1$ or $u^{q+1}=a$. If $u^{q-1}=1$ then

$$
\left(a^{k+1}\right)^{(q-1) / 2}=\left(u^{2(k+1)}\right)^{(q-1) / 2}=1 .
$$

But as $k+1$ is odd then $\left(a^{k+1}\right)^{(q-1) / 2}=-1$. Hence $u^{q-1} \neq 1$. If $u^{q+1}=a$ then

$$
\left(a^{k+1}\right)^{(q+1) / 2}=\left(u^{2(k+1)}\right)^{(q+1) / 2}=\left(u^{q+1}\right)^{k+1}=a^{k+1} .
$$

In this case, as $k+1$ is odd, $\left(a^{(q+1) / 2}\right)^{k+1}=\left(a^{(q-1) / 2} a\right)^{k+1}=-a^{k+1}$ and we again have a contradiction. Hence $f_{k}(X, a)$ has no roots in $\mathbb{F}_{q}$.

The next theorem is taken from [1].

THEOREM 2.3. [Chou] Let $q$ be odd and $k$ be a positive integer. Fix $a \in \mathbb{F}_{q}^{*}$ and let $b \in \mathbb{F}_{q^{2}}^{*}$ satisfy $b^{2}=a$. Set $e=1$ if $b \in \mathbb{F}_{q}$ and $e=2$ if $b \notin \mathbb{F}_{q}$. Write $k+1=p^{r}(m+1)$ with $(m+1, p)=1$ and $r \geqslant 0$. For each divisor $d>2$ of $2(m+1)$, let $n_{d}$ be the smallest integer satisfying $q^{n_{d}} \equiv \pm 1 \bmod d$. Then,

(1) If $f \in \mathbb{F}_{q}[X]$ satisfies: if $e=1$ then $f(X) \neq(X \pm 2 b)$ and if $e=2$ then $f(X) \neq$ $\left(X^{2}-4 a\right)$; then $f$ is an irreducible factor of $f_{m}(X, a)$ if and only if $f(X)$ is an irreducible factor of $f_{k}(X, a)$ of multiplicity $p^{r}$.

(2) if $e=1$ then $(X-2 b)$ and $(X+2 b)$ are irreducible factors of $f_{k}(X, a)$ of multiplicity $\left(p^{r}-1\right) / 2$, and if $e=2$ then $\left(X^{2}-4 a\right)$ is an irreducible factor of $f_{k}(X, a)$ of multiplicity $\left(p^{r}-1\right) / 2$,

(3) $f_{m}(X, a)$ is simple,

(4) $f_{m}(X, a)$ has the linear factor $X$ whenever $m$ is odd,

(5) for any divisor $d>4$ of $2(m+1)$ with $d \equiv 0 \bmod 4$,

(a) if $n_{d}$ is even, $n_{d} / 2$ is odd, $e=2$ and either $\left(d, q^{n_{d} / 2}-1\right)=d / 2$ or $\left(d, q^{n_{d} / 2}+1\right)=$ $d / 2$ then there are exactly $\phi(d) / n_{d}$ irreducible factors of $f_{m}(X, a)$ over $\mathbb{F}_{q}$ with degree $n_{d} / 2$ where every such factor is of the form

$$
f(x)=\prod_{i=0}^{n_{d} / 2-1}\left(x-b^{q^{i}}\left(\zeta_{d}+\zeta_{d}^{-1}\right)^{q^{i}}\right)
$$

where $\zeta_{d}$ is a primitive dth root of unity,

(b) otherwise, there are exactly $\phi(d) /\left(2 \operatorname{lcm}\left(e, n_{d}\right)\right)$ irreducible factors over $\mathbb{F}_{q}$ of $f_{m}(X, a)$ with degree $\mathrm{lcm}\left(e, n_{d}\right)$ and any such factor is of the form

$$
f(x)=\prod_{i=0}^{\operatorname{lcm}\left(e, n_{d}\right)-1}\left(x-b^{q^{i}}\left(\zeta_{d}+\zeta_{d}^{-1}\right)^{q^{i}}\right),
$$

where $\zeta_{d}$ is a primitive dth root of unity, 
(6) for any divisor $d>2$ of $2(m+1)$ with $d \not \equiv 0 \bmod 4$, if $d$ is even put $t=\phi(d) /\left(2 \operatorname{lcm}\left(e, n_{d}\right)\right)$ and if $d$ is odd put $t=(\phi(d)+\phi(2 d)) /\left(2 \mathrm{lcm}\left(e, n_{d}\right)\right)$. Then there are exactly $t$ irreducible factors of $f_{m}(X, a)$ of degree $1 \mathrm{~cm}\left(e, n_{d}\right)$ so that any such factor is of the form (2). Moreover, if $d>2$ is an odd divisor of $2(m+1)$, the set of all irreducible factors of $f_{m}(X, a)$ over $\mathbb{F}_{q}$ corresponding to $d$ equals the set of all irreducible factors of $f_{m}(X, a)$ over $\mathbb{F}_{q}$ corresponding to $2 d$.

We note that the next lemma is an extension of [2, Lemma 3] as it includes all square $a \in \mathbb{F}_{q}$.

Lemma 2.4. Let $\eta(a)=1$. If $f_{k}(X, a)$ permutes $\mathbb{F}_{q}$ then either

(i) $q \equiv \pm 3 \bmod 8$ and $\left(2(k+1), p\left(q^{2}-1\right)\right)=8$, or

(ii) $\left(k+1, p\left(q^{2}-1\right)\right)=2$.

Proof: As $f_{k}(X, a)$ permutes $\mathbb{F}_{q}$ then it has one linear factor. If $p$ divides $k+1$ then $\left(x^{2}-4 a\right)=(x+2 \sqrt{a})(x-2 \sqrt{a})$ divides $f_{k}(x, a)$. Therefore $(k+1, p)=1$ and in Theorem $2.3 k=m$. From (3) of Theorem $2.3 f_{k}(X, a)$ is simple so each of its factors has multiplicity one.

Put $D=\left(2(k+1), q^{2}-1\right)$. If $D=2$ then $\left(k+1, q^{2}-1\right)=1$ and from Lemma 2.1 $f_{k}(X, a)$ has no linear factors. Let $d>1$ be an odd prime divisor of $D$. As $(q-1, q+1)=2$ then $d$ divides one of $q-1$ or $q+1$ and $q \equiv \pm 1 \bmod d$. In Theorem $2.3 n_{d}=1$. From (6) of Theorem $2.3 f_{k}(X, a)$ has $(\phi(d)+\phi(2 d)) / 2>1$ linear factors over $\mathbb{F}_{q}$. This contradicts that $f_{k}(X, a)$ permutes $\mathbb{F}_{q}$, so $D=2^{r}$ where $r>1$.

Suppose that $d=2^{s}$, where $s>2$ is a divisor of $D$ and $q \equiv \pm 1 \bmod d$. Then from (5b) of Theorem $2.3 f_{k}(X, a)$ has $\phi(d) / 2>1$ distinct linear factors over $\mathbb{F}_{q}$. Again this contradicts the permutation property of $f_{k}(X, a)$, so $D=4$ and $\left(k+1, q^{2}-1\right)=2$ which establishes (ii).

Now suppose $q \not \equiv \pm 1 \bmod d$ for any $d=2^{s}$ dividing $D$ where $s>2$. In particular, $q \equiv \pm 3 \bmod 8$, so 8 is the highest power of 2 dividing $q^{2}-1$ and $\left(2(k+1), q^{2}-1\right)=8$. This is the condition in (i).

We could also have proven this lemma by combining [2, Lemma 3] and Lemma 1.2. [2, Lemma 4] can also be extended to all square $a \in \mathbb{F}_{q}$ by applying Lemma 1.2.

Lemma 2.5. If $f_{k}(X, a)$ permutes $\mathbb{F}_{q}$ and $\eta(a)=1$ then $\left(k(k+2), q^{2}-1\right)=1$ if $p=3$ and $\left(k(k+2), q^{2}-1\right)=3$ otherwise.

We have a result similar to Lemma 2.4 for non-square $a \in \mathbb{F}_{q}$.

Lemma 2.6. Let $\eta(a)=-1$. If $f_{k}(X, a)$ permutes $\mathbb{F}_{q}$ then either

(i) $q \equiv \pm 1 \bmod d$ for all $d>4$ dividing $\left(2(k+1), q^{2}-1\right)$ with $d \equiv 0 \bmod 4$, or

(ii) $\left(k+1, q^{2}-1\right)=2$.

Proof: As $f_{k}(x, a)$ permutes $\mathbb{F}_{q}$, it has exactly one linear factor. If $k+1$ is odd then from Lemma $2.2 f_{k}(X, a)$ has no roots in $\mathbb{F}_{q}$. Therefore $k+1$ is even and $X$ must be the 
only linear factor of $f_{k}(X, a)$ over $\mathbb{F}_{q}$. Put $k+1=p^{r}(m+1)$ where $(m+1, p)=1$. From (1) $f_{k}(X, a)=f_{m}^{p^{r}}(X, a)\left(X^{2}-4 a\right)^{\left(p^{r}-1\right) / 2}$ and we can consider linear factors of $f_{m}(X, a)$ instead. From Theorem 2.3 part $(3) f_{m}(X, a)$ is simple so each factor has multiplicity one.

Let $D=\left(2(m+1), q^{2}-1\right)$. We have $D \equiv 0 \bmod 4$. Let $d$ be a divisor of $D$ such that $d \equiv 0 \bmod 4$ and $d>4$. If $q \equiv \pm 1 \bmod d$ then in Theorem $2.3 n_{d}=1$ and there are no linear factors to be found, other then $X$. Part (i) now follows. If $q \not \equiv \pm 1 \bmod d$ then $n_{d}=2$ (as $d$ divides $q^{2}-1$ ) and there are $\phi(d) / 2$ linear factors over $\mathbb{F}_{q}$ of $f_{k}(X, a)$. But this contradicts the permutation property of $f_{k}(X, a)$ as $d>4$ so $\phi(d) / 2>1$. Therefore $D=4$ and $\left(k+1, q^{2}-1\right)=2$, establishing part (ii).

\section{LiNEAR FACTORS OF THE DPSK WHERE $q$ IS EVEN}

Throughout this section we assume $q$ is even. We have the following result which is analogous to Lemma 2.1. The proof is omitted as it is similar to the proof of Lemma 2.1.

LEMma 3.1. Let $q$ be even. Then $f_{k}(X, a)$ has no roots in $\mathbb{F}_{q}$ if and only if $\left(k+1,2\left(q^{2}-1\right)\right)=1$.

The following theorem is taken from [1].

ThEOREM 3.2. [Chou] Let $q$ be even and $k$ be a positive integer. Fix $a \in \mathbb{F}_{q}$ and let $b \in \mathbb{F}_{q}$ satisfy $b^{2}=a$. Write $k+1=2^{r}(m+1)$ where $m$ is even and $r \geqslant 0$. For each divisor $d>1$ of $m+1$ let $n_{d}$ be the smallest integer satisfying $q^{n_{d}} \equiv \pm 1 \bmod d$. Then,

(1) if $f \in \mathbb{F}_{q}[X]$ and $f(X) \neq X$, then $f$ is an irreducible factor of $f_{m}(X, a)$ if and only if $f$ is an irreducible factor of $f_{k}(X, a)$,

(2) for $m>0, f_{m}(X, a)=h(X)^{2}$ where $h(X)$ is simple and $h(0) \neq 0$,

(3) $X^{2^{r}-1}$ is a factor of $f_{k}(X, a)$ and any other irreducible factor of $f_{k}(X, a)$ has multiplicity $2^{r+1}$,

(4) for any divisor $d>1$ of $m+1$ there are exactly $\phi(d) /\left(2 n_{d}\right)$ irreducible factors of $f_{m}(X, a)$ over $\mathbb{F}_{q}$ with degree $n_{d}$ so that any such factor is of the form

$$
f(X)=\prod_{i=0}^{n_{d}-1}\left(x-b^{q^{i}}\left(\zeta_{d}+\zeta_{d}^{-1}\right)^{q^{i}}\right)
$$

where $\zeta_{d}$ is a primitive dth root of unity.

We have the following result which relies on the above theorem and is similar to Lemmas 2.4 and 2.6.

LEMmA 3.3. If $q$ is even and $f_{k}(X, a)$ permutes $\mathbb{F}_{q}$ then $\left(k+1, q^{2}-1\right)=3$ if $k+1$ is odd and $\left(k+1, q^{2}-1\right)=1$ if $k+1$ is even. 
Proof: As $f_{k}(X, a)$ permutes $\mathbb{F}_{q}$ it must have one linear factor over $\mathbb{F}_{q}$. Put $D=$ $\left(k+1, q^{2}-1\right)$ and suppose $D>1$. From part (3) of the previous theorem, $X$ is a factor of $f_{k}(X, a)$ if and only if $k+1$ is even. Suppose that $k+1$ is odd. Put $d_{1}=(k+1, q-1)$ and $d_{2}=(k+1, q+1)$. At least one of $d_{1}$ or $d_{2}$ must be greater then 1 . Now $q \equiv 1 \bmod d_{1}$ and $q \equiv-1 \bmod d_{2}$ which means $n_{d_{1}}=n_{d_{2}}=1$ in Theorem 3.2.

Suppose that $d_{1}>1$. Using part (4) of Theorem 3.2 we obtain $\phi\left(d_{1}\right) / 2$ distinct linear factors of $f_{k}(X, a)$ over $\mathbb{F}_{q}$. As $f_{k}(X, a)$ is a permutation polynomial of $\mathbb{F}_{q}$ then $\phi\left(d_{1}\right)=2$ which means $d_{1}=3,4$ or 6 . As $k+1$ and $q-1$ are odd then $d_{1}=3$. Similarly, if $d_{2}>1$ then $d_{2}=3$. As 3 may only divide one of $q-1$ or $q+1$ then we deduce exactly one of $d_{1}$ or $d_{2}$ must be 3 . Hence $D=3$.

If $k+1$ is even then $X$ is a factor of $f_{k}(X, a)$. Put $k+1=2^{r}(m+1)$ where $(m+1,2)=1$. From (1) $f_{k}(X, a)=X^{2^{r}-1} f_{m}^{2^{r}}(X, a)$. As $f_{k}(X, a)$ is a permutation polynomial $f_{m}(X, a)$ can have no linear factors, so from Lemma $3.1\left(m+1, q^{2}-1\right)=1$. [

We do not include a proof of our final result as it can be established in much the same way as Lemma 2.5, see [2, Lemma 4].

Lemma 3.4. Let $q$ be even and $f_{k}(X, a)$ permute $\mathbb{F}_{q}$. Then $\left(k(k+2), q^{2}-1\right)=1$ if $k+1$ is odd and $\left(k(k+2), q^{2}-1\right)=3$ if $k+1$ is even.

\section{REFERENCES}

[1] W.S. Chou, 'The factorization of Dickson polynomials over finite fields', Finite Fields Appl. 3 (1997), 84-96.

[2] S.D. Cohen, 'Dickson polynomials of the second kind that are permutations', Canad. J. Math 46 (1994), 225-238.

[3] S.D. Cohen, 'Dickson permutations', in Number-theoretic and algebraic methods in Computer Science (Moscow 1993) (World Scientific Publishing, River Edge, NJ, 1995), pp. 29-51.

[4] M. Henderson and R. Matthews, 'Permutation properties of Chebyshev polynomials of the second kind over a finite field', Finite Fields Appl. 1 (1995), 115-125.

[5] R. Lidl, G.L. Mullen and G. Turnwald, Dickson polynomials, Pitman Monographs and Surveys in Pure and Applied Maths 65 (Longman Scientific and Technical, Essex, England, 1993).

[6] R. Lidl and H. Niederreiter, Finite fields, Encyclopedia Math. Appl. 20 (Addison-Wesley, Reading, 1983), (now distributed by Cambridge University Press).

[7] R. Matthews, Permutation polynomials in one and several variables, Ph.D. Thesis (University of Tasmania, Tasmania, Australia, 1982).

School of Information Technology

The University of Queensland

Queensland 4072 Australia

e-mail: marie@it.uq.edu.au 